Ethnicity, social deprivation and psychological

\title{
distress in adolescents
}

\author{
School-based epidemiological study in east London
}

\begin{abstract}
STEPHEN A. STANSFELD, MARY M. HAINES, JENNY A. HEAD, KAMALDEEP BHUI, RUSSELL VINER, STEPHANIE J. C. TAYLOR, SHEILA HILLIER, EMILY KLINEBERG and ROBERT BOOY
\end{abstract}

\begin{abstract}
Background In adults the prevalence of psychological distress varies in different ethnic groups, and this has been explained by differences in socio-economic status. Is this also the case in adolescents?
\end{abstract}

Aims To examine whether ethnic differences in prevalence of psychological distress in adolescents are associated with social deprivation.

Method Across-sectional questionnaire survey was used to assess 2790 male and female pupils, aged II-14 years, from a representative sample of 28 east London secondary schools.

Results Rates of psychological distress were similar to rates in UK national samples in boys and girls. Bangladeshi pupils, although highly socially disadvantaged, had a lower risk of psychological distress $(\mathrm{OR}=0.63,95 \% \mathrm{Cl}$ 0.4-0.9). Non-UK White girls had higher rates of depressive symptoms ( $O R=1.54$, $95 \%$ Cl I.I-2.2).

Conclusions High rates of depressive symptoms in non-UK White girls may be related to recent migration. Low rates of psychological distress in Bangladeshi pupils in this sample relative to White pupils, despite socio-economic disadvantage, could be associated with cultural protective factors that require further investigation.

Declaration of interest None. Funding detailed in Acknowledgements.
Rates of psychological distress in adults vary according to ethnic group (Nazroo, 1997): higher levels are explained by social disadvantage, whereas lower rates are explained by culturally specific protective factors such as social cohesion (Nazroo, 1997; Sproston \& Nazroo, 2002). There are continuities between psychological distress in childhood, adolescence and adulthood, and there may be common risk and protective factors relating to ethnicity in childhood and adulthood (Harrington et al, 1990). Less is known about ethnicity and psychological distress in adolescents, in whom developmental factors, educational issues and concepts of 'youth culture' may modify the relationships of ethnicity and distress (Garratt, 1996). The 1999 British Child and Adolescent Mental Health Survey included too few Black and ethnic minority young people for an adequate exploration of the relationship between ethnicity and psychological distress (Meltzer $e t$ $a l, 2000)$. The aims of our study were first, to measure the prevalence of psychological distress in a representative, school-based sample of adolescents from different ethnic groups, and second, to assess whether ethnic differences in psychological distress were associated with social disadvantage.

\section{METHOD}

\section{Study design and sample selection}

The Research with East London Adolescents: Community Health Survey (RELACHS) is a school-based epidemiological study of a representative sample of 2790 adolescents from year 7 (11-12 years) and year 9 (13-14 years) attending 28 schools in east London in 2001. All 42 eligible schools in the boroughs of Hackney, Tower Hamlets and Newham were stratified by borough and school type (comprehensive, voluntary and other). Thirty schools were randomly selected and balanced to ensure representation of single-gender and mixed-gender schools; three declined and one was replaced. In each school that agreed to participate, representative mixed-ability classes were selected (two classes each from year 7 and year 9). The pupil response rate was $84 \%$ overall $(85 \%$ in non-Whites, $77 \%$ in Whites); $3.5 \%$ refused to participate and $12.2 \%$ were unavailable on the survey day, including $3.2 \%$ absent sick, $0.9 \%$ on holiday, $1.0 \%$ attending another activity and $7.1 \%$ absent without explanation.

\section{Ethnicity and cultural indicators}

Ethnicity was self-rated by the adolescents using an adaptation of the Census 2001 questions (Office for National Statistics, 2001). Black children included those who classified themselves as Black African, Black British, Black Somali and Black African-Caribbean; small numbers precluded analysing these groups individually. The 'mixed' ethnicity group included White and Black Caribbean; White and Black African; White and Black; White and Asian; and mixed 'other'. The 'other' group included Chinese and Vietnamese, among others. Country of birth, main languages spoken at home, religion and its observance, and length of time in the UK were collected by child self-report in the questionnaire.

\section{Socio-demographic factors}

Previous research suggests that the use of multiple indicators of socio-economic status collected from adolescents can reliably indicate household socio-economic status (Rogers et al, 1995; Health Education Authority, 1997). In the questionnaire, socio-economic indicators included parental employment status, household crowding (>1.5 persons/room) and family car ownership. In addition, eligibility for free school meals was obtained from school records. We selected eligibility for free school meals as our main socio-economic adjustment variable because it is known to be a reliable indicator of socio-economic status in UK studies of young people, and it was also the indicator on which we had the most complete data (Sammons et al, 1997).

\section{Main outcome measures}

Data were collected by questionnaire on mental and physical health, health behaviours, height, weight and sociodemographic factors. The following 
outcomes were included in the analyses reported in this paper.

\section{Mental health}

Child mental health was measured using the self-report Strengths and Difficulties Questionnaire (SDQ; Goodman, 1997; Goodman et al, 1998) which is a widely used psychometrically valid instrument for assessing psychological morbidity in UK children. A total difficulties score was derived by adding the scores of each of four sub-scales (emotional symptoms, conduct problems, peer problems and hyperactivity), producing a total score ranging from 0 to 40 . In a nationally representative sample of adolescents (aged 11-15 years), the 1999 British Child and Adolescent Mental Health Survey, the overall prevalence of psychiatric morbidity was $11.2 \%(12.8 \%$ boys, $9.6 \%$ girls aged $11-15$ years), established using multiple methods (Meltzer et al, 2000; http://www.sdqinfo.com). A score of 18 was chosen as the threshold for a 'high scorer' on the child self-completion version of the SDQ because this gave prevalence figures in the national data that were equivalent to the $9.5 \%$ prevalence found using multi-modal assessments (i.e. including data from parents and teachers) at national level (for children aged 5-15 years). We applied the same threshold to our data. The SDQ has been used in Bangladeshi adolescents before (Mullick \& Goodman, 2001) and thus has validity in this group. The SDQ was supplemented by the brief and reliable 13-item Short Moods and Feelings Questionnaire (SMFQ; Angold et al, 1995) to measure core depressive symptoms. A threshold of 8 or more was chosen to define high scorers. This threshold yielded a positive predictive value of $80 \%$ and a negative predictive value of $68 \%$ in the original validation against the Diagnostic Interview Schedule for Children - Depression Scale (Angold et al, 1995).

\section{Procedure}

\section{Data collection}

Copies of the questionnaire were group administered to the pupils, who completed them individually in classrooms under the supervision of researchers who answered pupils' questions and checked questionnaires for missing data. Parents were informed by letter of the study, and passive consent (with active opt-out) was used.
Pupils gave written consent to participation in the study. The study protocol was approved by the East London and the City Local Research Ethics Committee.

\section{Statistical analysis}

All analyses were weighted to take account of unequal probabilities of selection. As the sample selection used a stratified cluster design with pupils clustered within schools, standard errors and $95 \%$ confidence intervals for means and proportions were calculated using the survey estimation (svy) commands available in STATA (StataCorp, 1999).

Odds ratios and corresponding 95\% confidence intervals were calculated using the STATA logistic regression command with the cluster option specified. Three sets of logistic regression analyses were conducted:

(a) the proportions of children with high total SDQ and high SMFQ scores were compared by ethnic group;

(b) the proportions of children with high total SDQ and SMFQ scores were compared by socio-economic factors;

(c) a multivariate analysis including both ethnic group and socio-economic factors was performed.

These analyses were carried out for boys and girls combined and all models were adjusted for year group, gender and an interaction term between gender and year group. This interaction was included because the SDQ and SMFQ scores increased with age in girls but decreased in boys.

\section{RESULTS}

More than three-quarters of the participating children $(78 \%)$ were non-White; the majority $(79 \%)$ had been born in the UK and $9 \%$ had been in the UK for less than 5 years.

\section{Gender differences in psychological distress}

Both psychological distress and depressive symptoms increased with age in girls and decreased in boys (Table 1). In year 7 psychological distress scores were similar for boys and girls, but by year 9 boys had lower scores than girls. A similar pattern was seen for depressive symptoms, except that girls constituted a higher proportion of the cases at year 7 as well as year 9 (Table 1).

\section{Comparison with national data}

Scores on the SDQ in our cohort were compared with national data from the 1999 British Child and Adolescent Mental Health Survey (Meltzer et al, 2000) and the Health of Young People 1995-97 survey (Prescott-Clarke \& Primatesta, 1998). Based on the self-reported SDQ data from the 1999 survey and self-reported data in our survey, using the same threshold of 18 for high scorers in both studies, the proportion of high scorers on the SDQ was similar in boys in our sample $(9.3 \%, 95 \%$ CI $7-12)$ and in boys the 1999 British Child and Adolescent Mental Health Survey sample (9.4\% of boys aged 11-15 years). Rates were also similar for girls, with slightly higher rates of $11.3 \%$ (95\% CI 10-13) in our sample being SDQ high scorers, compared with $9 \%$ of girls in the 1999 survey (Meltzer et al, 2000). The proportion of high scorers among girls in our survey was higher than the proportion of high scorers among girls in the Health of Young People study ( $7 \%$ in girls aged $10-12$ years and $6 \%$ in girls aged $13-15$ years in the latter), although rates were similar in boys in both studies (11\% in boys aged $10-12$ years and $9 \%$ in boys aged 13-15 years in the Health of Young People survey; PrescottClarke \& Primatesta, 1998). In the Health of Young People study the SDQ was parent-reported rather than self-reported and the threshold for high scorers was 17.

\section{Ethnic differences in rates of psychological distress}

The largest ethnic groups were Bangladeshi $(25 \%)$, White UK $(21 \%)$ and Black $(20 \%)$. All ethnic groups experienced high levels of familial social disadvantage compared with the national average, but this was most evident in the Bangladeshi group (Table 2). Rates of psychological distress on the SDQ varied modestly by ethnic group (Table 3 ). This was similar for the SMFQ, except that higher rates of depressive symptoms on the SMFQ were found in non-UK White girls, who were largely of Irish, Turkish or Greek origin.

Contrary to our expectations, psychological distress and depressive symptoms had little consistent association with social disadvantage using the indices of parental unemployment, lack of car ownership, 
Table I Age and gender differences in psychological distress and depressive symptoms scores

\begin{tabular}{|c|c|c|c|}
\hline & Year 7 & Year 9 & Total \\
\hline \multicolumn{4}{|c|}{ Strengths and Difficulties Questionnaire } \\
\hline \multicolumn{4}{|l|}{ Boys } \\
\hline Score: mean (s.e.m.) & II.I (0.23) & $10.5(0.18)$ & $10.8(0.17)$ \\
\hline Case: $\%(95 \% \mathrm{Cl})$ & $10.5(8-14)$ & $8.0(6-10)$ & $9.3(7-12)$ \\
\hline \multicolumn{4}{|l|}{ Girls } \\
\hline Score: mean (s.e.m.) & $10.8(0.31)$ & II.3 (0.28) & II.I (0.23) \\
\hline Case: $\%(95 \% \mathrm{Cl})$ & $11.3(8-15)$ & $11.4(9-14)$ & $11.3(10-13)$ \\
\hline \multicolumn{4}{|l|}{ Bases (unweighted) } \\
\hline Boys & 675 & 655 & 1330 \\
\hline Girls & 675 & 740 & 1415 \\
\hline \multicolumn{4}{|c|}{ Short Moods and Feelings Questionnaire } \\
\hline \multicolumn{4}{|l|}{ Boys } \\
\hline Score: mean (s.e.m.) & $4.69(0.18)$ & $4.13(0.22)$ & $4.41(0.14)$ \\
\hline Case: $\%(95 \% \mathrm{Cl})$ & $21.6(19-25)$ & $16.4(13-21)$ & $18.9(16-22)$ \\
\hline \multicolumn{4}{|l|}{ Girls } \\
\hline Score: mean (s.e.m.) & $5.22(0.22)$ & $6.15(0.26)$ & $5.71(0.16)$ \\
\hline Case: $\%(95 \% \mathrm{Cl})$ & $26.3(23-30)$ & $32.9(29-37)$ & $29.8(27-33)$ \\
\hline \multicolumn{4}{|l|}{ Bases (unweighted) } \\
\hline Boys & 668 & 657 & 1325 \\
\hline Girls & 671 & 730 & $|40|$ \\
\hline
\end{tabular}

household overcrowding or eligibility for free school meals (Table 4).

Odds ratios for SDQ high scores were statistically significantly lower among Bangladeshi pupils compared with the White UK group adjusting for gender, year group and the interaction of gender and year group (Table 5). This lower risk remained after further adjustment for socioeconomic status in terms of eligibility for free school meals. The low risk of psychological distress in Bangladeshi pupils might be due to a preference for expressing psychological distress in a somatic idiom rather than through emotional expression. However, the rates of reported somatic symptoms did not differ by ethnicity.

Non-UK White pupils had a higher risk of depressive symptoms on the SMFQ than White UK pupils adjusting for gender, year group, the interaction of gender and year group and socio-economic status, and although not significant, the odds ratio for a high SDQ score was also raised in this group (Table 5). It seemed possible that this might relate to refugee status. We

Table 2 Ethnicity and socio-economic status of the study group

\begin{tabular}{|c|c|c|c|c|c|c|}
\hline Ethnicity & $\begin{array}{l}\text { Boys } \\
n(\%)\end{array}$ & $\begin{array}{l}\text { Girls } \\
n(\%)\end{array}$ & $\begin{array}{c}\text { No car } \\
\%(95 \% \mathrm{Cl})\end{array}$ & $\begin{array}{l}\text { Eligible for free } \\
\text { school meals' } \\
\%(95 \% \mathrm{Cl})\end{array}$ & $\begin{array}{l}\text { Overcrowded } \\
\%(95 \% \mathrm{Cl})\end{array}$ & $\begin{array}{c}\text { Parent } \\
\text { unemployed } \\
\%(95 \% \mathrm{Cl})\end{array}$ \\
\hline White UK & $284 \quad(2 I)$ & 297 (2I) & $28(23-35)$ & $39(32-47)$ & $12(9-15)$ & $26(2|-3|)$ \\
\hline White other & $73 \quad(5)$ & $88 \quad(6)$ & $34(27-4 I)$ & $58(49-67)$ & $20(14-26)$ & $45(34-56)$ \\
\hline Bangladeshi & $371 \quad(28)$ & $319 \quad(23)$ & $40(36-44)$ & $66(54-76)$ & $5 \mathrm{I}(46-57)$ & $62(59-66)$ \\
\hline Pakistani & 103 & $81 \quad(6)$ & $14(9-20)$ & $50(40-60)$ & 31 (24-39) & $35(26-44)$ \\
\hline Indian & $106 \quad(8)$ & $144(10)$ & $15(10-24)$ & $34(24-45)$ & $21(15-28)$ & $26(19-35)$ \\
\hline Black & 248 (18) & $327 \quad(23)$ & $30(26-34)$ & $40(33-48)$ & $23(19-28)$ & $25(19-32)$ \\
\hline Mixed & $97 \quad(7)$ & $97 \quad(7)$ & $39(32-48)$ & $46(36-56)$ & $17(12-24)$ & $30(24-38)$ \\
\hline Other & $59 \quad(4)$ & $56 \quad(4)$ & $18(\mathrm{II}-27)$ & $44(28-60)$ & $16(9-28)$ & $29(18-44)$ \\
\hline All & $|34|(100)$ & $1409(100)$ & $30(28-33)$ & $48(42-54)$ & $28(23-33)$ & $37(32-43)$ \\
\hline
\end{tabular}

I. In England $14.9 \%$ and in London $24.5 \%$ of secondary school pupils are eligible for free school meals (Department for Education and Skills, 2002).

examined the year of entering the UK as a marker for recent migration and possible refugee status or asylum-seeking. Overall, $9 \%$ of pupils had entered the UK in the previous 5 years, but this proportion was higher in non-UK White pupils $(26 \%)$ and Black pupils (19\%). There was poorer mental health among more recent entrants than among the rest of the sample. Among nonUK White pupils, adjustment for recent migration reduced the risk of psychological distress on the SDQ from $1.36(95 \% \mathrm{CI}$ $0.8-2.3)$ to 1.19 (95\% CI 0.7-2.0) and on the SMFQ from 1.54 (95\% CI 1.1-2.2) to 1.25 (95\% CI 0.9-1.8). Another possibility was that household composition might explain the high risk of psychological distress. We found that rates of psychological distress were similar in pupils from two-parent and mother-only families, and that the increased risk of depressive symptoms in the non-White UK pupils was not explained by differences between single-parent or two-parent household composition (results not reported).

Eligibility for free school meals was not associated with increased risk of a high SDQ score or with risk of depressive symptoms after adjustment for ethnicity. This weak association of socio-economic status with SDQ and SMFQ did not appear to differ by ethnic group (significance of interaction term for eligibility for free school meals by ethnic group, $P=0.52$ ).

\section{DISCUSSION}

This is the largest UK study of psychological distress in a representative sample of adolescents from ethnic minority groups. Psychological distress increased with age in girls and decreased in boys, in keeping with other studies (Meltzer et al, 2000). The rates of psychological distress in our study were similar to national data. Bangladeshi pupils, although highly socially disadvantaged, had a lower risk of psychological distress than White UK pupils. Non-UK White girls had higher rates of depressive symptoms than White UK girls.

\section{Socio-economic position and psychological distress}

Somewhat surprisingly, there was no association between indices of social disadvantage and psychological distress. In contrast, national data in the Health of Young People study showed a social gradient of high scores in parent-reported SDQ 
Table 3 Analysis of high scorers on the Strengths and Difficulties Questionnaire (SDQ) and the Short Moods and Feelings Questionnaire (SMFQ): gender and ethnicity

\begin{tabular}{|c|c|c|c|c|}
\hline \multirow[t]{3}{*}{ Ethnicity } & \multicolumn{2}{|c|}{ SDQ case' } & \multicolumn{2}{|c|}{ SMFQ case ${ }^{2}$} \\
\hline & Boys & Girls & Boys & Girls \\
\hline & $\%(95 \% \mathrm{Cl})$ & $\%(95 \% \mathrm{Cl})$ & $\%(95 \% \mathrm{Cl})$ & $\%(95 \% \mathrm{Cl})$ \\
\hline White UK & $10.3(7-16)$ & I3.8 (II-17) & $17.9(13-24)$ & $29.7(24-36)$ \\
\hline White other & $14.0(8-23)$ & I4.I (6-30) & $16.9(8-34)$ & $4 I .4(3 I-52)$ \\
\hline Bangladeshi & 5.7 (4-9) & $9.7(8-12)$ & $19.0(14-24)$ & $26.6(20-34)$ \\
\hline Pakistani & $7.6(4-15)$ & $14.9(6-31)$ & 18.7 (15-24) & $27.8(15-46)$ \\
\hline Indian & $12.7(5-27)$ & $10.5(8-13)$ & 19.2 (12-29) & $28.6(20-38)$ \\
\hline Black & $9.2(6-13)$ & $10.9(8-14)$ & $17.2(12-23)$ & $29.5(25-34)$ \\
\hline Mixed & $17.0(10-27)$ & 8.9 (4-19) & $26.0(18-37)$ & $29.1(19-40)$ \\
\hline Other & $8.4(5-14)$ & $6.7(3-16)$ & $22.1(14-34)$ & $3 I .4(23-4 I)$ \\
\hline
\end{tabular}

I. Score of 18 or more on the SDQ.

2. Score of 8 or more on the SMFQ.

by social class of head of household (Prescott-Clarke \& Primatesta, 1998). The lack of effect of socio-economic position within our cohort may reflect lack of variability within it, given that every ward in the three boroughs lies in the bottom quintile of the government's index for deprivation (Department of Environment, Transport and the Regions, 2000). Measures of socio-economic position may not be equally indicative of social disadvantage across ethnic groups (Davey Smith, 2000), although we found a lack of association across all ethnic groups, including White
UK pupils. Lack of variation in socioeconomic status and the possibility of area effects on psychological distress, even for those of more advantaged social position, may explain the apparently small effects of social disadvantage. Child psychiatric disorder also showed no social gradient related to poverty among Native Americans in the Great Smoky Mountains Study, although there was such a gradient in White children (Costello et al, 1997). The authors argued that the effects of poverty might have been mitigated by protective aspects of community or family life.

Table 4 Analysis of high scorers on the Strengths and Difficulties Questionnaire (SDQ) and the Short Moods and Feelings Questionnaire (SMFQ): gender and socio-economic status

\begin{tabular}{|c|c|c|c|c|}
\hline \multirow[t]{3}{*}{ Socio-economic factor } & \multicolumn{2}{|c|}{ SDQ case ${ }^{l}$} & \multicolumn{2}{|c|}{ SMFQ case $^{2}$} \\
\hline & Boys & Girls & Boys & Girls \\
\hline & $\%(95 \% \mathrm{Cl})$ & $\%(95 \% \mathrm{Cl})$ & $\%(95 \% \mathrm{Cl})$ & $\%(95 \% \mathrm{Cl})$ \\
\hline \multirow{2}{*}{\multicolumn{5}{|c|}{$\begin{array}{l}\text { Eligibility for free school } \\
\text { meals }\end{array}$}} \\
\hline & & & & \\
\hline No & $8.4(7-11)$ & $11.7(9-15)$ & $18.2(15-22)$ & $30.6(28-34)$ \\
\hline Yes & $9.9(7-13)$ & $10.9(9-13)$ & $19.4(17-23)$ & $29.2(25-34)$ \\
\hline \multicolumn{5}{|l|}{ Car ownership } \\
\hline 0 & $10.6(8-14)$ & $9.4(7-13)$ & $20.8(16-26)$ & $28.5(25-32)$ \\
\hline I & $7.9(6-11)$ & $11.9(10-15)$ & $17.9(15-21)$ & $30.7(27-34)$ \\
\hline $2+$ & $10.4(7-15)$ & $11.9(9-16)$ & $18.1(14-23)$ & $29.0(23-36)$ \\
\hline \multicolumn{5}{|l|}{ Overcrowding } \\
\hline$<\mathrm{l} .5$ persons/room & $8.8(7-I I)$ & $11.3(10-13)$ & $17.8(15-21)$ & $29.3(27-32)$ \\
\hline$\geqslant 1.5$ persons/room & $10.2(7-15)$ & $10.5(9-13)$ & $21.9(19-27)$ & $30.8(25-37)$ \\
\hline \multicolumn{5}{|l|}{ Parental employment } \\
\hline One/both working & $9.2(7-12)$ & II.3 (9-14) & $18.1(15-21)$ & $29.6(27-33)$ \\
\hline Neither working & $9.4(7-13)$ & $11.4(9-14)$ & $20.0(15-26)$ & $30.2(26-35)$ \\
\hline
\end{tabular}

I. Score of 18 or more on the SDQ.

2. Score of 8 or more on the SMFQ.

\section{Limitations of the study}

The SDQ is a well-accepted screening instrument for adolescent mental ill-health, and the self-report version has been validated in a community and clinic sample (Goodman et al, 1998). In many studies it is used in a multi-method version incorporating parental and teacher versions of the questionnaire, to provide an overall prevalence score using an algorithm. The major limitation of our study was the reliance on self-reported assessment of psychological distress without triangulation by parental and teacher accounts and validation by a psychiatric assessment (Goodman et al, 2000). The sensitivity of the self-report version of the SDQ is low in other samples $(15.9 \%)$, although it is slightly higher for depressive disorders $(33.3 \%)$ and anxiety disorders $(22.1 \%)$. Thus, we might not have identified all cases of psychological distress. Moreover, we did not assess the validity of the thresholds for psychological distress on the SDQ or SMFQ in this study population. In unravelling differences in rates of psychological distress between ethnic minorities, it would be very informative to have standardised interview-based measures of psychological distress. This is a goal for this study in future data collection.

Part of the difference in rates of high SDQ scores between the Health of Young People survey and our study in girls may be methodological. In the former survey parents completed the SDQ on behalf of their children, and might have underreported problems (Prescott-Clarke \& Primatesta, 1998). In our survey the pupils completed their own SDQ, which might have led to higher rates of reporting problems. Self-reports of emotional disorders with the SDQ in children aged 11 years upwards are as useful as teacher data, but not as useful as parent data, and are of less predictive value for conduct and hyperactivity disorders than parent or teacher data (Goodman et al, 2000).

The cross-sectional nature of our study means we cannot be certain of the direction of association. Having insufficient numbers in the various Black categories meant merging Black children from very different cultures into a single group. There were also relatively small numbers in the Pakista$\mathrm{ni}$ and Indian groups. This was a further limitation, and more research is needed to examine psychological distress in these groups. Although the study lacked 
Table 5 Odds ratios for poor mental health: analysis by ethnic group and socio-economic status

\begin{tabular}{|c|c|c|c|c|}
\hline & \multicolumn{2}{|c|}{ SDQ case $(n=2491)$} & \multicolumn{2}{|c|}{ SMFQ case $(n=2475)$} \\
\hline & $\begin{array}{c}\text { Adjusted odds ratio' } \\
\text { OR }(95 \% \mathrm{Cl})\end{array}$ & $\begin{array}{l}\text { Plus adjustment for (a) ethnicity/ } \\
\text { (b) eligibility for free school meals } \\
\text { OR }(95 \% \mathrm{Cl})\end{array}$ & $\begin{array}{l}\text { Adjusted odds ratio' } \\
\text { OR }(95 \% \mathrm{Cl})\end{array}$ & $\begin{array}{c}\text { Plus adjustment for (a) ethnicity/ } \\
\text { (b) eligibility for free school meals } \\
\text { OR }(95 \% \mathrm{Cl})\end{array}$ \\
\hline \multicolumn{5}{|l|}{ (a) Ethnicity } \\
\hline White other & $1.39(0.8-2.3)$ & $1.36(0.8-2.3)$ & $1.53(I .1-2.2)$ & $1.54(I .1-2.2)$ \\
\hline Bangladeshi & $0.64(0.4-0.9)$ & $0.63(0.4-0.9)$ & $0.92(0.7-1.3)$ & $0.92(0.7-1.3)$ \\
\hline Pakistani & $0.92(0.4-2.1)$ & $0.91(0.4-2.1)$ & $0.97(0.6-1.6)$ & $0.97(0.6-1.6)$ \\
\hline Indian & $1.02(0.7-1.6)$ & $\mathrm{I} .03(0.7-\mathrm{I} .6)$ & $1.0 \mathrm{I}(0.7-\mathrm{I} .5)$ & $\mathrm{I} .0 \mathrm{I}(0.7-\mathrm{I} .5)$ \\
\hline Black & $0.89(0.6-1.3)$ & $0.89(0.6-1.3)$ & $0.94(0.7-1.2)$ & $0.94(0.7-1.3)$ \\
\hline Mixed & $1.16(0.7-2.0)$ & $\mathrm{I} .15(0.7-\mathrm{I} .9)$ & $1.25(0.8-1.9)$ & $1.25(0.8-1.9)$ \\
\hline \multicolumn{5}{|c|}{ (b) Eligibility for free school } \\
\hline \multicolumn{5}{|l|}{ meals } \\
\hline No & 1 & 1 & 1 & 1 \\
\hline Yes & $\mathrm{I} .03(0.8-\mathrm{I} .4)$ & $1.10(0.8-1.5)$ & $0.98(0.8-1.2)$ & $0.99(0.8-1.2)$ \\
\hline
\end{tabular}

SDQ, Strengths and Difficulties Questionnaire; SMFQ, Short Moods and Feelings Questionnaire.

I. Adjusted for gender, year group and interaction term for gender $\times$ year group.

sufficient power to detect differences between the smaller ethnic groups, this was not the case for the Bangladeshi and White UK groups. As $12 \%$ of pupils were absent at the time of data collection, and as rates of psychological distress might have been higher among absentees, our estimates of psychological distress may underestimate the true prevalence. In general, there was a high rate of absence from schools, much of which could not be easily accounted for.

\section{Depressive symptoms in non-UK White pupils}

Non-UK White pupils had a higher risk of depressive symptoms relative to White UK pupils. This increased risk was diminished after adjustment for recent migration, suggesting that the excess risk for psychological distress might relate to stressors associated with migration. These might include traumatic experiences prior to migration, separation from parents and friends and difficulties adjusting to a new, alien environment (Bhugra \& Jones, 2001).

\section{Psychological distress in Bangladeshi pupils}

Despite their high levels of social disadvantage, Bangladeshi pupils relative to White UK pupils were at decreased risk of psychological distress on the SDQ even after adjustment for gender, year group, the interaction of gender and year group, and socio-economic status. Lower rates of psychological distress were also found in Bangladeshi girls (but not boys) in the British Child and Adolescent Mental Health Survey (Meltzer et al, 2000), suggesting that this is not an isolated finding. Why should Bangladeshi children have lower rates of psychological distress, when they live in conditions of social deprivation and Bangladeshi adults have high rates of physical illness (Bhopal et al, 1999)? One explanation could be that instruments such as the SDQ do not identify the idioms of psychological distress as effectively in Bangladeshi pupils as they do in pupils from other ethnic groups (Bhui et al, 2003). However, the SDQ has been used successfully to identify psychological distress in children in Bangladesh (Mullick \& Goodman, 2001), and we did not find higher rates of somatic symptoms in Bangladeshi pupils who might have had a tendency to somatise emotional symptoms, as has been suggested in south Asian adults (Rack, 1982). High levels of family support and high ethnic density in the east London Bangladeshi population may be protective factors for mental health, but further research is needed to explore this (Halpern \& Nazroo, 2000; Sproston \& Nazroo, 2002). Research is also needed on cultural identity, the impact of religious belief and observance and the impact of culture on health behaviour in relation to psychological distress.
It may be that the mental health effects of living in an area with high levels of deprivation are counterbalanced by ethnically related protective factors (Costello et al, 1997). In east London these factors might include high levels of family support, religious belief, strong cultural identity and cohesion. If any of these factors are protective of mental health in the face of social adversity, there may be implications for the prevention of psychological distress in adolescents.

\section{ACKNOWLEDGEMENTS}

The study was commissioned by East London and The City Health Authority to inform their Health Action Zone and we thank them for their support. We thank Tower Hamlets, City \& Hackney and Newham Primary Care Trusts for additional funding. S.J.C.T. is funded by a National Career Scientist Award in Public Health from the Department of Health. We are grateful for the support of the schools, parents and students involved in this study. We also thank the field team, including Wendy Isenwater, Giash Ahmed, Sarah Brentnall, Sultana Choudry-Dormer and Franca Davenport.

\section{REFERENCES}

Angold, A., Costello, E. J., Messer, C. J., et al (1995) Development of a short questionnaire for use in epidemiological studies of depression in children and adolescents. International Journal of Methods in Psychiatric Research, 5, 237-249.

Bhopal, R., Unwin, N., White, M., et al (1999) Heterogeneity of coronary heart disease risk factors in 
Indian, Pakistani, Bangladeshi, and European origin populations: cross sectional study. BMJ, 319, 215-220.

Bhugra, D. \& Jones, P. (200I) Migration and mental illness. Advances in Psychiatric Treatment, 7, 216-222.

Bhui, K., Mohamud, S., Warfa, N., et al (2003) Cultural adaptation of mental health measures: improving the quality of clinical practice and research. British Journal of Psychiatry, 183, 184-186.

Costello, E. J., Farmer, E. M., Angold, A., et al (1997) Psychiatric disorders among American Indian and White Youth in Appalachia: The Great Smoky Mountains Study American journal of Public Health, 87, 827-832.

Davey Smith, G. (2000) Learning to live with complexity: ethnicity, socioeconomic position and health in Britain and the United States. American Journal of Public Health, 90, 1694-1698.

Department for Education and Skills (2002) Statistics of Education Schools in England, 2000 Edition. London: DFES. Available at http: / /www.dfes.gov.uk/ rsgateway/DB/VOL/v000359/index.shtml

Department of Environment, Transport and the Regions (2000) Measuring Multiple Deprivation at the Small Area Level: The Indices of Deprivation 2000. London: Department for Environment, Food and Rural Affairs.

Garratt, D. (1996) Youth cultures and sub-cultures. In Youth in Society (eds J. Roche \& S. Tucker), pp. 143-150. London: Sage.

Goodman, R. (1997) The Strengths and Difficulties Questionnaire: a research note. Journal of Child Psychology and Psychiatry, 38, 58I-586.

Goodman, R., Meltzer, H. \& Bailey, V. (1998) The Strengths and Difficulties Questionnaire: a pilot study on the validity of the self-report version. European Child and Adolescent Psychiatry, 7, 125-130.

Goodman, R., Ford, T., Simmons, H., et al (2000) Using the Strengths and Difficulties Questionnaire (SDQ) to screen for child psychiatric disorders in a community sample. British Journal of Psychiatry, 177 $534-539$.

Halpern, D. \& Nazroo, J. (2000) The ethnic density effect: results from a national community survey of England and Wales. International Journal of Social Psychiatry, 46, 34-46.

Harrington, R., Fudge, H., Rutter, M., et al (1990) Adult outcomes of childhood and adolescent depression. Archives of General Psychiatry, 47, 465-473.

Health Education Authority (1997) Young People and Health: Health Behaviour in School-Aged Children. London: HEA

Meltzer, H., Gatward, R., Goodman, R., et al (2000) Mental Health of Children and Adolescents in Great Britain London: Stationery Office.

Mullick, M. S. \& Goodman, R. (200I) Questionnaire screening for mental health problems in Bangladeshi children: a preliminary study. Social Psychiatry and Psychiatric Epidemiology, 36, 94-99.

Nazroo, J.Y. (1997) Ethnicity and Mental Health London: Policy Studies Institute.

Office for National Statistics (2001) Census 2001. http://www.statistics.gov.uk/census200l/census_ background.qs.asp

Prescott-Clarke, P. \& Primatesta, P. (1998) Health Survey for England: The Health of Young People 95-97. London: Stationery Office.

\section{CLINICAL IMPLICATIONS}

- This study is further evidence that local services need to know their community well and be able to interpret local needs.

- Health promotion strategies that aim to reduce psychological distress in adolescents should focus on understanding the full range of potential protective factors, including cultural factors.

- Recent migration may be a risk factor for depressive symptoms in adolescent girls.

\section{LIMITATIONS}

- The main outcome was a self-reported assessment of psychological distress, which could be strengthened by validation using a psychiatric interview.

- The data reported were cross-sectional, which meant that the direction of association between ethnicity, social deprivation and psychological distress could not be determined.

- Small sample sizes within the different Black ethnic groups meant that variation in psychological distress within these different cultural groups could not be reliably assessed.

STEPHEN A. STANSFELD, PhD, MRCPsych, MARY M. HAINES, PhD, Department of Psychiatry, Institute of Community Health Sciences, Barts and The London, Queen Mary's School of Medicine and Dentistry, University of London; JENNYA. HEAD, MSc, Department of Psychiatry, Institute of Community Health Sciences, Barts and The London, Queen Mary's School of Medicine and Dentistry, University of London, and Department of Epidemiology and Public Health, University College London and the Royal Free Medical School, London; KAMALDEEP BHUI, MD, MRCPsych, Department of Psychiatry, Institute of Community Health Sciences, Barts and The London, Queen Mary's School of Medicine and Dentistry, University of London; RUSSELL VINER, PhD, Department of Medicine, Royal Free and University College London Medical School; STEPHANIE J.C. TAYLOR, MFPHM, MRCGP, MD, Department of General Practice and Primary Care, Institute of Community Health Sciences, Barts and The London, Queen Mary's School of Medicine and Dentistry, University of London; SHEILA HILLIER, PhD, Department of Human Science and Medical Ethics, Institute of Community Health Sciences, Barts and The London, Queen Mary's School of Medicine and Dentistry, University of London; EMILY KLINEBERG, BPsychol, Department of Psychiatry, Institute of Community Health Sciences, Barts and The London, Queen Mary's School of Medicine and Dentistry, University of London; ROBERT BOOY, MRCPCH, MD, Department of Health, Institute of Community Health Sciences, Barts and The London, Queen Mary's School of Medicine and Dentistry, University of London, London UK

Correspondence: Professor Stephen Stansfeld, Department of Psychiatry, Barts and The London, Queen Mary's School of Medicine and Dentistry, Queen Mary, University of London, Medical Sciences Building, Mile End Road, London El 4NS, UK. Tel: +44 (0)207 882 7727; fax: +44 (0)207 8827924 ; e-mail: s.a.stansfeld@qmul.ac.uk

(First received 10 October 2003, final revision 24 March 2004, accepted 31 March 2004)

Rack, P. (1982) Culture and Mental Disorder. London: Tavistock.

Rogers, A., Karlsen, S., McCarthy, M., et al (1995) Camden and Islington Young People and Health Project. Survey of Health Behaviours and Attitudes in 993 15-YearOlds in C \& I Schools. London: Department of Epidemiology and Public Health, University College London Medical School.
Sammons, P., West, A. \& Hind, A. (1997) Accounting for variation in pupil attainment at the end of Key Stage I. British Educational Research Journal, 23, 489-5II.

Sproston, K. \& Nazroo, J. (eds) (2002) Ethnic Minority Psychiatric Illness Rates in the Community (EMPIRIC). London: Stationery Office.

StataCorp (1999) Stata Statistical Software: Release 6.0. College Station, TX: Stata Corporation. 